\title{
Dados químicos e farmacológicos sobre as plantas utilizadas como medicinais pela comunidade do bairro Ponta Grossa, Porto Alegre, Rio Grande do Sul
}

\author{
Giovana Secretti Vendruscolo ${ }^{1}$, Stela Maris K. Rates ${ }^{2}$, Lilian Auler Mentz ${ }^{1 *}$ \\ ${ }^{1}$ Departamento de Botânica, Instituto de Biociências, Universidade Federal do Rio Grande do Sul, 91509-900, \\ Porto Alegre, Rio Grande do Sul, Brasil, \\ ${ }^{2}$ Departamento de Produção de Matéria-Prima, Faculdade de Farmácia, Universidade Federal do Rio Grande do \\ Sul, 90610-000, Porto Alegre, RS, Brasil
}

\begin{abstract}
RESUMO: A partir do levantamento das plantas utilizadas como medicinais por moradores e Agentes Comunitários de Saúde, do Posto de Saúde da Família do bairro Ponta Grossa, Porto Alegre, Rio Grande do Sul, foram selecionadas as dez espécies mais importantes para a comunidade. Para estas espécies foi realizada uma revisão bibliográfica, buscando estudos sobre sua constituição química e atividade farmacológica. Para todas as espécies foram encontrados dados químicos e para nove delas dados de atividade farmacológica. Foram encontrados estudos clínicos para quatro espécies e usos terapêuticos aprovados por organismos internacionais para três espécies. Efeitos adversos foram encontrados, na literatura, para cinco das dez espécies pesquisadas e para seis destas, o uso durante a gestação é desaconselhado.
\end{abstract}

Unitermos: Etnobotânica, plantas medicinais, farmacologia.

\begin{abstract}
Chemical and pharmacologic data on medicinal plants used by the community of the Ponta Grossa neighborhood, Porto Alegre, Rio Grande do Sul, Brazil”. The ten most important species among all plants used as medicinal by the residents and Communitary Agents of Health from the Family Health Office of Ponta Grossa neighborhood, Porto Alegre, Rio Grande do Sul, Brazil were selected. A bibliographical review for these species was accomplished, finding studies about the chemical constitution for all species and some pharmacologic activity for nine of them. Clinical studies for four species and therapeutic uses approved by international organisms for three species were found. It was verified that according to the literature, adverse effects for five of the ten researched species and for six of these the use is dissuaded during the gestation.
\end{abstract}

Keywords: Ethnobotany, medicinal plants, pharmacology.

\section{INTRODUÇÃO}

No Brasil, a possibilidade de implementação da fitoterapia no sistema público de saúde vem sendo considerada desde 1988 (CIPLAN) e faz parte das diretrizes da I Conferência Nacional de Assistência Farmacêutica (CNMAF, 2003). A partir de então, várias inicativas pontuais para estabelecer a fitoterapia na rede pública de sáude vêm ocorrendo, com destaque para o Programa de Fitoterapia do Ceará, alicerçado no Projeto Farmácias Vivas, idealizado pelo Prof. Francisco José de Abreu Matos (Matos et al., 2001). No entanto, para a maioria das plantas nativas não existem estudos científicos e o uso no Brasil é baseado principalmente na tradicionalidade. A OMS reconhece a importância do uso tradicional, mas para a utilização de uma planta com finalidade terapêutica, em nível de saúde pública, é fundamental o estabelecimento de sua segurança, eficácia e garantia de qualidade das preparações (Lapa et al., 2003; WHO, 2002; Rates, 2001). O uso inadequado destes recursos terapêuticos pode originar efeitos adversos retardados e/ou assintomáticos, interações medicamentosas ainda não estudadas e dificilmente reconhecidas, além de retardar o diagnóstico e tratamento apropriado (Cañigueral; Vila, 2003; Rates, 2001). Nestes termos, o emprego no atendimento primário à saúde pode gerar um impacto social (Lapa et al., 2003), com aumento de gastos nos serviços públicos de saúde, visto que as plantas com maior volume de estudos científicos que garantam eficácia e segurança constituem matéria-prima importada, o que torna urgente a busca de informações científicas sobre as espécies utilizadas na medicina popular.

Assim, os objetivos deste trabalho foram revisar os dados químicos e farmacológicos publicados em literatura científica e os usos terapêuticos aprovados por organismos internacionais, para as dez espécies utilizadas como medicinais, selecionadas por Vendruscolo (2004) como as mais importantes para os moradores e Agentes Comunitários de Saúde no bairro Ponta Grossa, Porto 
Alegre, Rio Grande do Sul. Este levantamento servirá de base futura para a elaboração de um manual que será devolvido como retorno para a comunidade estudada, visando fornecer à população e aos profissionais de saúde subsídios para a utilização racional destes recursos, respeitando o contexto da tradicionalidade.

\section{MATERIAL E MÉTODOS}

Vendruscolo (2004) realizou um levantamento das plantas utilizadas como medicinais por 51 moradores do bairro Ponta Grossa, Porto Alegre, Rio Grande do Sul. A partir deste levantamento constatou-se a utilização de 150 espécies pertencentes a 59 famílias. Em um segundo trabalho, Vendruscolo (2004) utilizou um teste estatístico para selecionar as dez espécies consideradas mais importantes para a população estudada, através do índice de Valor de Uso, segundo a metodologia de Phillips e Gentry (1993).

Para as dez espécies com maior índice de Valor de Uso foi realizada uma revisão bibliográfica, utilizando como palavras chave o binômio científico, nas bases de dados Biological Abstracts e Medline, sem restrição de anos. Sempre que possível, os artigos completos foram consultados e quando estes não eram acessíveis, foram utilizadas as informações constantes nos resumos. Livros didáticos e de divulgação científica da área de farmacognosia também foram utilizados, entre os quais Simões et al. (2003) e Bruneton (1993). Resumos publicados em congressos ou outras reuniões científicas não foram considerados, exceto quando não encontradas outras informações na literatura consultada.

Para o item "Usos terapêuticos aprovados" foram consultadas algumas obras bibliográficas com dados sobre eficácia e segurança, recomendadas como referência pelo Ministério da Saúde (Brasil, 2004), entre as quais estão Newall et al. (2002), Blumenthal et al. (2000), Gruenwald et al. (2000), Blumenthal (1998), ESCOP (1997) e Germosén-Robineau (1997), além de outras obras que apresentam monografias elaboradas por especialistas (Der Marderosian, 2001; Tyler, 1994).

\section{RESULTADOS}

Para nove das dez espécies pesquisadas foram encontrados dados químicos e/ou farmacológicos experimentais, e apenas para quatro delas, estudos clínicos. Este último fato poderia ser justificado por motivos éticos e de segurança, já que estudos clínicos somente são permitidos após uma extensa avaliação pré-clínica (Lapa et al., 2003). Para todas as espécies são conhecidos os constituintes químicos principais, os quais nem sempre estão relacionados com as atividades farmacológicas relatadas. Três espécies têm usos terapêuticos aprovados.

A seguir são apresentadas as dez espécies, em ordem decrescente de Valor de Uso, segundo Vendruscolo
(2004), com as informações de dados químicos e farmacológicos encontrados. Na Tabela 1 são referidos o nome científico válido, seguido dos sinônimos quando relevantes, família, nome(s) popular(es), usos tradicionais, parte utilizada, forma de preparo, usos terapêuticos aprovados e efeitos adversos. Sempre que possível, os usos foram mencionados conforme as citações dos informantes, mas em alguns casos foram sumarizados pelos autores.

\section{Aloe arborescens Mill. (Asphodelaceae) -}

Constituintes químicos: Látex das folhas com derivados antracênicos: aloína, barbaloína e isobarbaloína (Kuzuya et al., 2001), aloe-emodina, aloenina (Ali et al., 1999); a porção interna da folha contém mucilagem (Wozniewski et al., 1990) e glicanos (Hikino et al., 1986). Dados farmacológicos: Derivados antracênicos têm ação laxativa (Kuzuya et al., 2001; Quing et al., 1991). O gel composto por mucilagem possui propriedades emoliente, hidratante, antiinflamatória e antibacteriana (Tyler, 1994). Extratos etanólico, clorofórmico, benzênico e aquoso de folhas secas e frescas apresentam ação antifúngica (Ali et al., 1999). O suco das folhas e os polissacarídeos isolados têm ação hipoglicemiante (Beppu et al., 1993). Müller et al. (1996) demonstraram atividade genotóxica para alguns derivados antracênicos.

2. Citrus x aurantium L. (Rutaceae) - Constituintes químicos: Espécies do gênero Citrus são ricas em flavonóides, óleos voláteis, cumarinas e pectinas (Kuster; Rocha, 2003). A maioria dos compostos flavônicos são heterosídeos de flavanonas (hesperidosídeo, neohesperidosídeo, naringosídeo, eriodictiosídeo). Ocorrem também outros flavonóides, como a diosmina e o rutosídeo (Bruneton, 1993). A hesperidina é o principal glicosídio encontrado nas laranjas doces e a neoesperidina, nas laranjas amargas (Arriaga; Rumbero, 1990). O fruto imaturo contém sinefrina e n-metiltiramina (Huang et al., 1995). Dados farmacológicos: O óleo volátil da casca do fruto apresentou atividade sedativa/hipnótica, contrastando com o extrato etanólico das folhas, que não apresentou esta atividade (Carvalho-Freitas; Costa, 2002). O extrato alcoólico da casca dos frutos mostrou efeito antiespasmódico (Foster et al., 1980). Os frutos se mostraram, in vitro, potentes inibidores da atividade do rotavírus, que causa diarréias (Hyun et al., 2000). O suco dos frutos apresentou, in vitro, atividade antimicrobiana (Caceres et al., 1987). O extrato do fruto, em ratos, produziu uma redução do consumo de alimento e do ganho de peso corporal e um índice significativo de mortalidade. Este efeito foi atribuído à atividade $\beta$-adrenérgica da sinefrina (Calapai et al., 1999). Esta substância também está correlacionada com uma ação redutora da pressão portal, possivelmente através de uma vasoconstrição arterial, detectada na infusão da casca do fruto (Huang et al., 1995). 


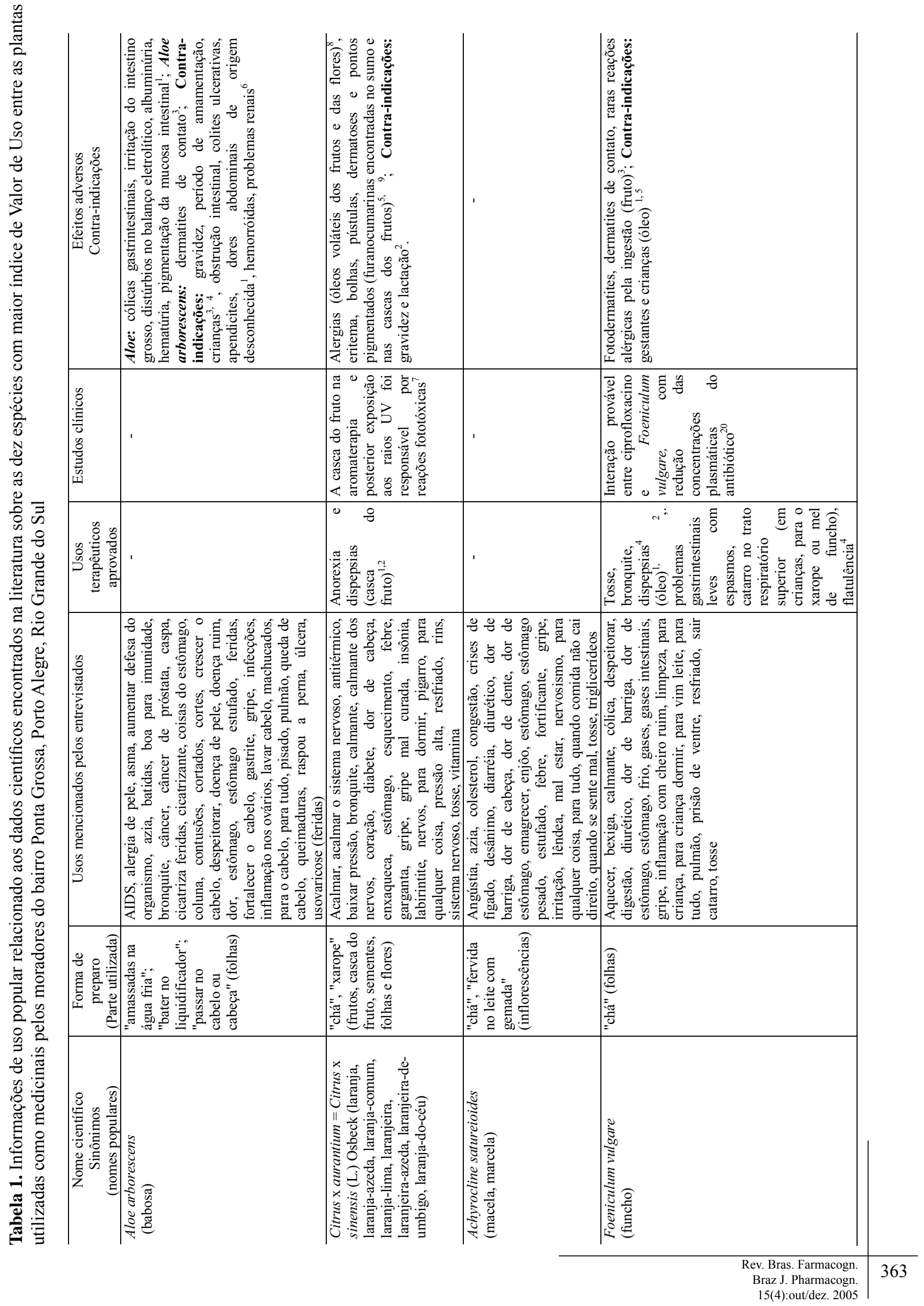




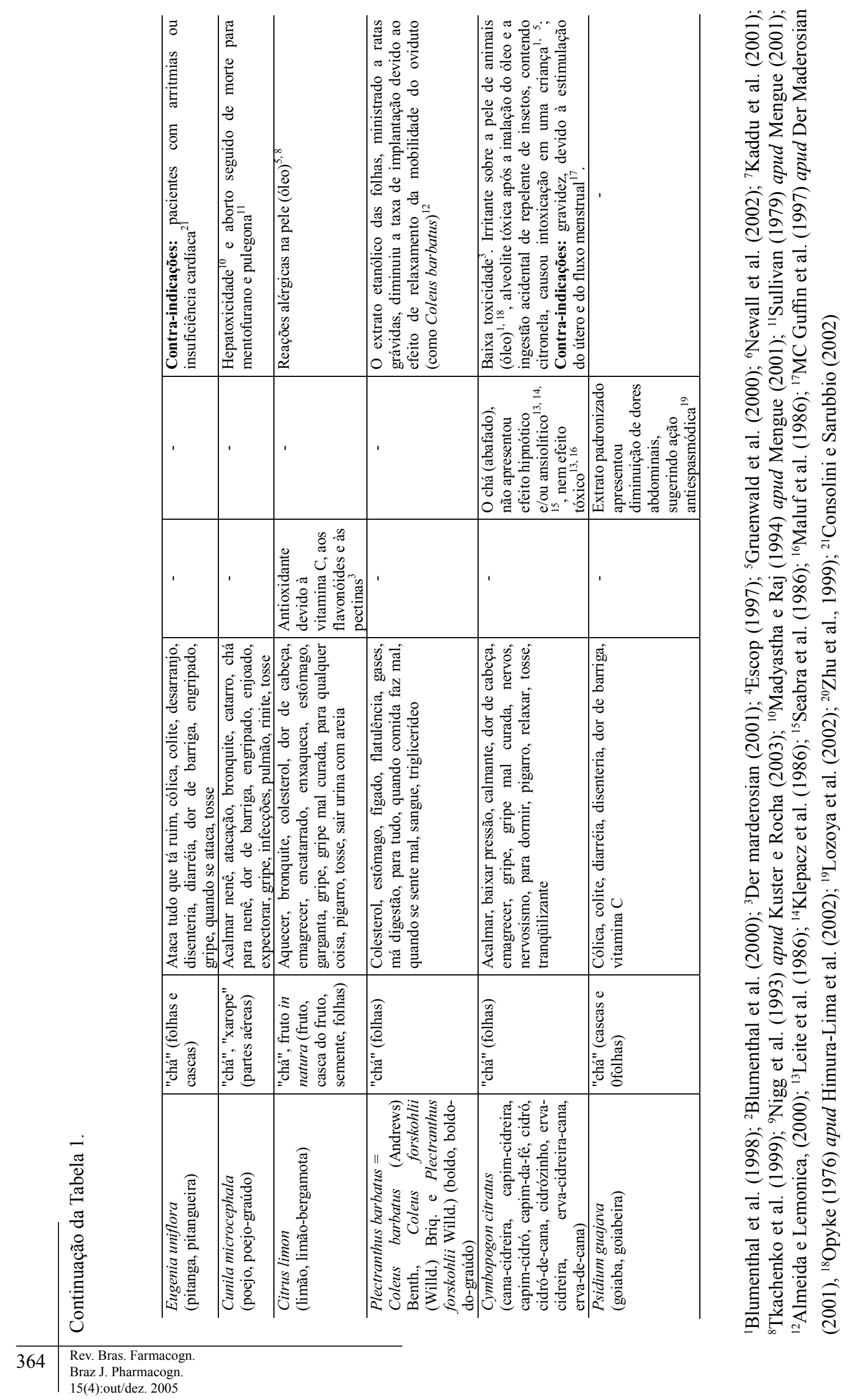




\section{Achyrocline satureioides (Lam.) DC. (Asteraceae)}

- Constituintes químicos: Contém ácido caféico (Desmarchelier et al., 1998; Simões, 1984; Ferraro et al., 1981), ácido clorogênico e isoclorogênico (Desmarchelier et al., 1998), derivados da fenilpirona e cavapirona (Kaloga et al., 1983), flavonóides (Desmarchelier et al., 1998; Mesquita et al., 1986; Simões, 1984), minerais (Puhlmann et al., 1992; Bassani, 1990), óleos voláteis (Lorenzo et al., 2000) e polissacarídeos (Puhlmann et al., 1992; Wagner et al., 1985). Para revisão, ver Petrovick et al. (1997). Dados farmacológicos: Diferentes extratos (aquoso, etanólico e hidroalcóolico) e frações purificadas (polissacarídeos e flavonóides) das inflorescências, folhas e caules têm sido extensivamente estudados, in vitro e in vivo, por diferentes autores, apresentando resultados promissores para as seguintes atividades: antiinflamatória, antiespasmódica e analgésica (Santos, 1996; Silva, 1993; Simões et al., 1988, 1986; Simões, 1988, 1984; Langeloh; Schenkel, 1985), colerética (Kadarian et al., 2002), sedativa (Simões et al., 1988, 1986; Simões, 1984), imunoestimulante (Puhlmann et al., 1992; Simões et al., 1986; Wagner et al.; 1985), antiviral (Simões, 1992), antimicrobiana (Gutkind et al., 1981; Mota, 1963), hipoglicemiante (Gugliucci; Menini, 2002a) e antioxidante (Gugliucci; Menini, 2002b; Souza, 2002; Desmarchelier et al., 1998).

4. Foeniculum vulgare Mill. (Apiaceae) - Constituintes químicos: Nos frutos são encontrados esteróis, triterpenos, flavonóides, cumarinas e óleo volátil (Tanira et al., 1996), constituído, principalmente, de anetol, fenchona e estragol (metil chavicol) (Zhu et al., 1999). O óleo das folhas possui estragol, alfa-felandreno, limoneno e fenchona (Garcia et al., 2000). Dados farmacológicos: O óleo volátil apresenta ação antioxidante (Oktay et al., 2003; Ruberto et al., 2000), hepatoprotetora (Ozbek et al., 2003), espasmolítica (Amjad, 2000), antibacteriana (Daswani; Bohra, 2002; Ruberto et al.; 2000) e antifúngica (Ezzat, 2001). Extratos aquoso e etanólico dos frutos mostraram atividade antioxidante (Oktay et al., 2003), diurética (Beaux et al., 1997; Tanira et al., 1996), analgésica, antipirética (Tanira et al., 1996), colagoga e antibacteriana (Daswani; Bohra, 2002; Tanira et al., 1996), sem apresentar toxicidade ou efeito genotóxico, em camundongos (Shah et al., 1991). Atividade diurética também foi demonstrada para o extrato hidroalcoólico das raízes (Beaux et al., 1997). O extrato aquoso de folhas é hipotensor, em ratos, provavelmente devido às ações diurética e natriurética (Bardai et al., 2001; Abdul-Ghani; Amin, 1988). O extrato acetônico dos frutos causou aumento no peso das glândulas mamárias, ovidutos, endométrio, miométrio, cervix e vagina, em ratas, indicando atividade estrogênica (Malini et al., 1985).

5. Eugenia uniflora L. (Myrtaceae) - Constituintes químicos: As folhas são ricas em taninos (Lee et al., 1997), glicosídeos deflavonóides (Schmeda-Hirschmann, 1995)e

óleo volátil (Santos; Mello, 2003). Dados farmacológicos: Extratos polares (aquoso e hidroalcóolico) das folhas causaram redução da secreção intestinal e da propulsão gastrintestinal (Almeida et al., 1995), efeito relaxante da aorta toráxica em ratos (Wazlawik et al., 1997), efeito diurético e hipotensor, mediados por uma ação vasodilatadora (Consolini; Sarubbio, 2002; Consolini et al., 1999) e baixa toxicidade aguda em camundongos (Schmeda-Hirschmann et al.; 1987). Flavonóides isolados das folhas inibiram a atividade da enzima xantino-oxidase (Schmeda-Hirschmann et al., 1987). O extrato metanólico das folhas apresentou ação antimalárica em camundongos (Agbedahunsi; Aladesanmi, 1993) e diversos extratos da folha apresentaram atividade tripanosomicida in vitro (Adewumni et al., 2001). Alguns autores encontraram atividade antimicrobiana para o óleo volátil (Holtez et al., 2002; Adebajo et al., 1989), enquanto outros não a encontraram (Schapoval et al., 1994).

\section{Cunila microcephala Benth. (Lamiaceae)}

- Constituintes químicos: O óleo volátil contém, principalmente, mentofurano e tujeno, além de traços de pulegona (Bordignon et al., 1997). Dados farmacológicos: Não foram encontrados estudos farmacológicos para esta espécie.

7. Citrus limon (L.) Osbeck (Rutaceae) - Constituintes químicos: $\mathrm{O}$ óleo volátil da casca do fruto e das folhas de Citrus limon é rico em limoneno, $\beta$-pineno, $\gamma$ terpineno, terpinoleno, neral e geranial (Vekiari et al., 2002; Bisset; Wichtl, 2001; Ghelardini et al., 1999). Dados farmacológicos: O óleo volátil causou inibição no crescimento de Candida albicans (Ezzat, 2001).

\section{Plectranthus barbatus Andrews (Lamiaceae) -} Constituintes químicos: Diterpenos, como forscolina (Bhat et al., 1977 - como Coleus forskohlii Willd.), coleolonol (Dubey et al., 1981 - como Coleus forskohlii), barbatusol (Kelecon, 1983 - como Plectranthus barbatus) e epi-desoxicolenol para as raízes (Tadon et al., 1992 - como Coleus forskohlii). Dados farmacológicos: Os extratos hexânico e metanólico de folhas, caules e raízes apresentaram atividades antibacteriana e antiinflamatória (Matu; Staden, 2003 - como Plectranthus barbatus). $\mathrm{O}$ extrato metanólico mostrou atividade relaxante do músculo liso traqueal (Kasonia, 1995). O coleonol mostrou efeito hipotensor e uma boa absorção no trato intestinal (Dubey et al., 1981- como Coleus forskohlii). A forscolina apresenta ação broncodilatadora e acentuada atividade inotrópica e vasodilatadora, relacionada à estimulação da enzima adenilciclase (Barreiro; Fraga, 2001).

9. Cymbopogon citratus (DC.) Stapf (Poaceae) - Constituintes químicos: As partes aéreas contêm flavonóides, alcalóides, saponinas, triterpenos, taninos e óleo volátil, rico em citral e mirceno (Matouschek; Stahl- 
Biskup, 1991; Sousa et al., 1991; Olaniyi et al., 1975). Dados farmacológicos: Extratos aquoso e etanólico das folhas, obtidos de diferentes formas, apresentam efeito antinociceptivo (Lorenzetti et al., 1991; Costa et al., 1989), hipotensor, diurético e antiinflamatório (Carbajal et al., 1989). Por outro lado, o abafado das folhas (extrato aquoso) se mostrou inativo em testes de atividade antipirética, ansiolítica, movimento do trânsito intestinal, coordenação e atividade motoras, anticonvulsivante, neuroléptica e controle da temperatura corporal, em roedores (Carlini et al., 1986; Contar et al., 1986), bem como não apresentou efeito tóxico sobre o processo reprodutivo, influência sobre o desenvolvimento da prole e efeitos sobre a glicemia, temperatura, peso corporal e comportamento, quando administrado por período prolongado a ratos machos e fêmeas (Formigoni et al., 1986). O extrato etanólico não apresentou atividade mutagênica no teste de Ames, com e sem ativação metabólica (Vinitketkumnuen et al., 1994). Cheah et al. (2001) demonstraram atividade antioxidante, in vitro, dos extratos diclorometano e metanólico. O óleo volátil e alguns terpenos isolados, mostraram ação antinociceptiva (Viana et al., 2000; Lorenzetti et al., 1991), antibacteriana (Cimanga et al., 2002; El-Kamali et al., 1998; Onawumni et al., 1984), inclusive contra Helicobacter pylori (Tomoyuki et al., 2003) e antifúngica contra cepas clínicas de Trichophyton mentagrophytes, Trichophyton rubrum, Epidermophyton floccosum e Microsporum gypseum (Wannissorn et al., 1996).

10. Psidium guajava L. (Myrtaceae) - Constituintes químicos: A casca do caule e as folhas contêm taninos (Santos; Mello, 2003; Okuda et al., 1982). As folhas contêm óleo volátil, álcoois sesquiterpênicos, flavonóides e ácidos triterpenóides (Santos; Mello, 2003). No extrato dosfrutos foram detectados monoterpenose sesquiterpenos (Wilson; Shaw, 1978). Dados farmacológicos: Diferentes extratos polares (aquoso e alcóolico) das folhas apresentam efeito antidiarréico (Lin et al., 2002; Olajide et al., 1999; Lutterodt; Almeida et al., 1995; Lutterodt, 1992; Maleque, 1988), antipirético e antiinflamatório (Olajide et al., 1999), inotrópico negativo (Garcia et al., 2003) e redutor da atividade locomotora exploratória espontânea de camundongos (Lutterodt; Maleque, 1988). O extrato aquoso das folhas apresentou efeito antitussígeno em porcos-da-índia, devido a uma ação relaxante da traquéia, mediada pelo sistema colinérgico, não se mostrando tóxico para roedores (Jaiarj et al., 1999). Extratos aquoso, hidroalcóolico, etanólico e metanólico de cascas e folhas foram testados, in vitro, com resultados promissores, para a atividade antibacteriana (Abdelrahim et al., 2002; Carvalho et al., 2002; Holtez et al., 2002; Vieira et al., 2001; Gnan; Demello, 1999; Martinez et al., 1997; Caceres et al., 1991; 1990), antifúngica (Holtez et al., 2002) e contra Entamoeba histolytica (Tona et al., 1998). Contudo, Martinez et al. (1997) não encontraram atividade contra Candida albicans e Guevara et al. (1994) contra Vibrio cholerae. O óleo volátil das folhas, raízes e flores, apresentou, in vitro, atividade antibacteriana (Fratini et al., 1991), atribuída à quercetina e seus glicosídeos (Grosvenor et al., 1995). Os extratos hexânico, acetato de etila e metanólico apresentaram efeito antinociceptivo dose-dependente (Shaheen et al., 2000). Sesquiterpenos isolados do extrato hexânico demonstraram efeito depressor do Sistema Nervoso Central, em camundongos. O extrato hexânico possui atividade relaxante da musculatura lisa, atribuída à atividade antagonista de cálcio apresentada pelo óxido de cariofileno (Meckes et al., 1996). A espécie foi alvo de revisão e estudo pelo Projeto Tramil (GermosenRobineau, 1997).

\section{DISCUSSÃO}

Observando-se os dados encontrados na literatura foi constatada uma grande carência de estudos que propiciem o uso seguro destas plantas pela população estudada. A maioria dos estudos são pré-clínicos, muitas vezes preliminares e in vitro ou in vivo. Somente foram encontrados estudos clínicos para Citrus $x$ aurantium, Cymbopogon citratus, Foeniculum vulgare e Psidium guajava. Mesmo para as plantas aprovadas pela Comissão E (Blumenthal et al., 1998; 2000) e outros órgãos regulatórios, não foram encontrados relatos de ensaios clínicos na revisão realizada. As espécies de uso aprovado por organismos internacionais são Citrus $\mathrm{x}$ aurantium, Foeniculum vulgare e Citrus limon. Para todas estas, as mesmas referências mencionam também restrições no uso. Além delas, outras duas espécies mais utilizadas foram alvo de investigação pelo Programa de Pesquisa em Plantas Medicinais, promovido pela Central de Medicamentos do Ministério da Previdência e Assistência Social do Brasil (Cymbopogon citratus) (Brasil, 1986) e pelo Projeto Tramil (Psidium guajava) (Germosen-Robineau, 1997).

Entre as dez plantas mais importantes para a comunidade do bairro, sete são introduzidas de diferentes regiões da América ou de outros continentes e três são nativas (Achyrocline satureioides, Cunila microcephala e Eugenia uniflora). Para Cunila microcephala foi encontrada somente uma referência de dados químicos, não sendo encontrados dados farmacológicos, ressaltando a carência de estudos com espécies nativas.

Os constituintes químicos presentes nas espécies geralmente são bastante variáveis, tanto do ponto de vista qualitativo quanto quantitativo, sendo que diferentes locais de coleta, secagem e diferentes formas de preparo das plantas e seus produtos dificultam a reprodutibilidade dos trabalhos (Farias, 2003). Além disto, diversos tipos de extratos são utilizados nos testes para diferentes ações biológicas, gerando, conseqüentemente, resultados diferentes. Também é necessário ressaltar que os extratos mencionados nos testes, geralmente, são diferentes da forma de preparo utilizada pela população. 
A grande maioria das espécies aqui enfocadas (nove) apresenta óleos voláteis entre seus componentes químicos principais. Este resultado poderia sugerir que as pessoas entrevistadas selecionam as espécies utilizadas pelo aroma e sabor, característicos das espécies que contêm óleos voláteis. Alguns constituintes dos óleos voláteis, presentes nas espécies aqui mencionadas, são relatados na literatura como potencialmente tóxicos, como o estragol (em quatro espécies), a tujona e cânfora (em duas espécies cada), o apiol e o mentofurano (em uma espécie cada).

Para cinco espécies foram encontrados relatos de efeitos adversos na literatura consultada. Estas são: Aloe arborescens, Citrus x aurantium, Citrus limon, Cymbopogon citratus, Foeniculum vulgare e Plectranthus barbatus. Também foram levados em conta efeitos adversos relatados para substâncias isoladas, como é o caso de Cunila microcephala, para a qual não existem relatos de toxicidade, mas que contém substâncias tóxicas, quando avaliadas isoladamente (Tabela 1). Contra-indicações ao uso foram encontadas para Aloe arborescens, Citrus x aurantium, Cymbopogon citratus, Foeniculum vulgare e Eugenia uniflora. Somente para Achyrocline satureioides e Psidium guajava não foram relatados na literatura consultada efeitos adversos e/ou contra-indicações.

Seis das dez plantas estudadas têm seu uso desaconselhado durante a gravidez. São elas: Aloe arborescens, Citrus x aurantium, Cunila microcephala, Cymbopogon citratus, Foeniculum vulgare e Plectranthus barbatus. Para as demais espécies esta informação não está disponível.

Concluindo, os dados aqui apresentados mostram que muitas das espécies utilizadas no bairro Ponta Grossa coincidem com as espécies utilizadas em outras comunidades do estado do Rio Grande do Sul (Marodin et al., 2003; Ritter et al., 2002; Mengue et al., 2001 e Marodin, 2000), o que expressa a extensão de seu uso popular. No entanto, nossos resultados, e também dos autores citados, mostram que a maioria dos dados científicos publicados provêm de trabalhos preliminares e que as atividades biológicas descritas nem sempre podem ser relacionadas com as indicações de uso popular. Por fim, este trabalho ratifica a importância do intercâmbio entre o conhecimento científico e o conhecimento popular como instrumento para a promoção do uso racional das plantas medicinais, bem como para a seleção de plantas para a implementação de investimentos em pesquisa e desenvolvimento, com vistas à implantação da Fitoterapia nos Serviços Públicos, com garantia de eficácia, segurança e qualidade.

\section{AGRADECIMENTOS}

Aos funcionários do Posto de Saúde da Família de Ponta Grossa e aos moradores do bairro Ponta Grossa, Porto Alegre, Rio Grande do Sul. Aos funcionários do herbário ICN e das bibliotecas do Departamento de Botânica e da Faculdade de Farmácia da UFRGS pela ajuda prestada.

\section{REFERÊNCIAS}

Abdelrahim SI, Almagboul AZ, Omer MEA, Elegami A 2002. Antimicrobial activity of Psidium guajava L. Fitoterapia 73: 713-715.

Abdul-Ghani AS, Amin R 1988. The vascular action of aqueous extracts of Foeniculum vulgare leaves. $J$ Ethnopharmacol 24: 213-218.

Adebajo AC, Oloke KJ, Aladesanmi AJ 1989. Antimicrobial activities and microbial transformation of volatile oils of Eugenia uniflora. Fitoterapia 50: 451-455.

Adewumni CO, Agbedahunsi JM, Adebajo AC, Aladesanmi AJ, Murphy N, Wando J 2001. Ethno-veterinary medicine: screening of Nigerian medicinal plants for trypanocidal properties. J Ethnopharmacol 77: 19-24.

Agbedahunsi JM, Aladesanmi AJ 1993. Effect of Eugenia uniflora on early malaria infection. Fitoterapia 54: 174-175.

Ali MIA, Shalaby NMM, Elgamal MHA, Mousa ASM 1999. Antifungal effects of different plant extracts and their major components of selected Aloe species. Phytother Res 13: 40-407.

Almeida CE, Karnikowski MGO, Foleto R, Baldisserotto B 1995. Analysis of antidiarrhoeic effect of plants used in popular medicine. Revista de Saúde Pública 29: 428-433.

Almeida FCG, Lemonica IP 2000. The toxic effects of Coleus barbatus $\mathrm{B}$. on the different periods of pregnancy in rats. J Ethnopharmacol 73: 53-60.

Amjad H 2000. Foeniculum vulgare therapy in irritable bowel syndrome. AJG Setember.

Arriaga EJ, Rumbero A 1990. Naringin, hesperidin and heohesperidin content in juices from thirteen Citrus spp. Fitoterapia 51: 31-36.

Bardai ES, Lyoussi B, Wibo M, Morel N 2001. Pharmacological evidence of hypotensive activity of Marrubium vulgare e Foeniculum vulgare in spontaneously hipertensive rat. Clin Exp Hypertens 23: 329-343.

Bassani VL 1990. Valorisation de formes galéniques vegétales. Desalcoolisation et concentration des solutions extractives sur membrane d'osmose inverse. Monyprllier Tese de Doutorado - University of Montpllier.

Beaux D, Fleurentin J, Mortier F 1997. Diuretic action of hydroalcohol extracts of Foeniculum vulgare var dulce (D.C.) roots in rats. Phytother Res 11: 320322.

Beppu H, Nagamura Y, Fujita K 1993. Hypoglycaemic and antidiabetic effects in mice of Aloe aroborescens Miller var. natalensis Berger. Phytother Res 7: $37-$ 42, Suplemento.

Bhat SV, Bajwa BS, Dornaur H, Souza NJ, Fehlhaber HW 1977. Structure and stereochemistry of new labdane diterpenoids from Coleus forskohlii Briq. Tetrahedron Lett. 19: 1669-1672.

Bisset NM, Wichtl M 2001. Herbal drugs and phytopharmaceuticals. 2.ed. Medpharm: Boca 
Raton, CRC / Stuttgart.

Blumenthal, M 1998. The complete German commission and monographs: Therapeutic guide to herbalmedicines. Austin, Texas: American Botanical Council.

Blumenthal M, Goldberg A, Brinkmann J 2000. Herbal medicine - Expanded commission and monographs. Newton, MA: Integrative medicine Communications.

Bordignon SAL, Schenkel EP, Spitzer V 1997. The essential oil composition of Cunila microcephala and Cunila fasciculata. Phytochemistry 44: 1283-1286.

Brasil, Ministério da Previdência e Assistência Social, Central de Medicamentos 1986. Considerações gerais sobre o uso do capim-cidró (Cymbopogon citratus (DC.) Stapf) em medicina popular. Brasília: CEME.

Brasil. Ministério da Saúde 2004. Agencia Nacional de Vigilância Sanitária. Lista de referências bibliográficas para avalaiação de segurança e eficácia de fitoterápicos. Resolução RE no 88 de 16 de março de 2004.

Bruneton J 1993. Pharmacognosie, phytochimie, plantes medicinales. 2.ed. Paris: Lavoisier.

Caceres A, Giron LM, Alvarado SR, Torres MF 1987. Screening of antimicrobial activity of plants popularly used in Guatemala for the treatment of dermatomucosal disiases. J Ethnopharmacol 20: 223-237.

Caceres A, Cano O, Samayoa B, Aguilar L 1990. Plants used in Guatemala for the treatment of gastrointestinal disorders. 1. Screening of 84 plants against enterobacteria. J Ethnopharmacol 30: 55-73.

Caceres A, Alvarez AV, Ovando AE, Samayoa BE 1991. Plants used in Guatemala for the treatment of respiratory disease. 1.Screening of 68 plants against grampositive bacteria. J Ethnopharmacol 31: 193-208.

Calapai G, Firenzuoli F, Saitta A, Squadrito F, Arlotta MR, Costantino G, Inferrera G 1999. Antiobesity and cardiovascular toxic effects of Citrus aurantium extracts in the rat: a preliminary report. Fitoterapia 70: 86-92.

Cañigueral S, Vila R 2003. La fitoterapia racional. In: Vanaclocha BV, Folcará SC (org.) Fitoterapia. Vademécum de prescripción.4.ed. Barcelona: Masson, p.15-27.

Carbajal D, Casaco A, Arruzazabala L, Gonzalez R, Tolon Z 1989. Pharmacological study of Cymbopogon citratus leaves. J Ethnopharmacol 25: 103-107.

Carlini EA, Contar JDP, Silva-Filho AR, Silveira-Filho NG, Frochtengarten ML, Bueno OFA 1986. Pharmacology of lemongrass (Cymbopogon citratus Stapf). I. Effects of teas prepared from the leaves on laboratory animals. J Ethnopharmacol 17: 37-64.

Carvalho AAT, Sampaio MCC, Sampaio FC, Melo AFM, Sena KXFR, Chiappeta AA, Higino JS 2002. Atividade antimicrobiana in vitro de extratos hidroalcóolicos de Psidium guajava L. sobre bácterias Gramnegativas. Acta Farmacéutica Bonariense 21: 255258.

Carvalho-Freitas MIR 2002.Costa M Anxiolytic and sedative effects of extracts and essential oil from Citrus aurantium L. Biol Pharm Bull 25: 1629-1633.

Cheah PB, Ng CH, Wong CF 2001. Antioxidant activity of tropical lemon grass (Cymbopogon citratus) extracts in linoleic acid and chicken fat systems. J Food Sci Tec 38: 62-64.

Cimanga K, Kambu K, Tona L, Apers S, Bruyne T, Hermans N, Totte J, Pieters L, Vlietinck AJ 2002. Correlation between chemical composition and antibacterial activity of essential oils of some aromatic medicinal plants growing in the Democratic Republic of Congo. J Ethnopharmacol 79: 213-220.

CNMAF. Conferência Nacional de Medicamentos e Assistência Farmacêutica 1. 2003. Relatório final preliminar. Brasília: Ministério da Saúde.

Consolini AE, Baldini OAN, Amat AG 1999. Pharmacological basis for the empirical use of Eugenia uniflora L. (Myrtaceae) as antihypertensive. J Ethnopharmacol 66: 33-39.

Consolini AE, Sarubbio MG 2002. Pharmacological effects of Eugenia uniflora (Myrtaceae) aqueous crude extract on rats heart. J Ethnopharmacol 81: 57-63.

Contar JDE, Ferreira TMS, Souza MLO, Lodder HM, Silva-Filho AR, Bueno OFA, Carlini EA 1986. Farmacologia pré-clínica do capim-cidrão (Cymbopogon citratus Stapf). Ensaios psicofarmacológicos preliminares. In: Brasil, Ministério da Previdência e Assistência Social, Central de Medicamentos (org.) Considerações gerais sobre o uso do capim-cidró (Cymbopogon citratus (DC.) Stapf em medicina popular. Brasília: CEME, p.13-24.

Costa M, Di Stasi LC, Kirizawa M, Mendaçolli SLJ, Gomes C, Trolin G 1989. Screening in mice of some medicinal plants used for analgesic purposes in the state of São Paulo. Part II. J Ethnopharmacol 27: 25-33.

Daswani L, Bohra A 2002. Antibacterial effects of fennel (Foeniculum vulgare) on the growth of human pathogenic strain of Staphylococcus aureus. Advances in Plant Sciences 15: 589-596.

Der Marderosian A 2001. The review of natural products: The most complete source of natural products information. Saint Louis: Facts and Comparisons.

Desmarchelier C, Coussio J, Ciccia G 1998. Antioxidant and free radical scavenging effects in extracts of the medicinal herb Achyrocline satureioides (Lam.) DC. ("marcela"). Braz J Med Biol Res 31: 11631170.

Dubey MP, Srimal RC, Nityanand S, Dhawan BN 1981. Pharmacological studies on coleonol, a hypotensive diterpne from Coleus forskohlii. J Ethnopharmacol 3: 1-13.

El-Kamali HH, Ahmed AH, Mohammed AS, Yahia AAM, ElTayeb LH, Ali AA 1998. Antibacterial properties of essential oils from Nigella sativa seeds, Cymbopogon citratus leaves e Pulicaria undulata aerial parts. Fitoterapia 59: 77-78.

ESCOP - European Scientific Cooperative on Phytotherapy. 1997. Monographs on the medicinal uses of plant drugs. Exeter: Centre for Complementary Health Studies.

Ezzat SM 2001. In vitro inhibition of Candida albicans growth by plant extracts and essential oils. World J Microb Biot 17: 757-759.

Farias M 2003. Avaliação da qualidade de matérias-primas vegetais. In: Simões CMO, Shenkel EP, GOSMANN G, Mello JCP, Mentz LA, Petrovick PR (org.) Farmacognosia: da planta ao medicamento. 5.ed. Porto Alegre/Florianópolis: Editora da UFRGS/ Editora da UFSC, p.262-288.

Ferraro GE, Norberto C, Coussio JD 1981. Polyphenols from Achyrocline satureioides. Phytochemistry 20: 2053- 
2054.

Formigoni MLOS, Lodder HM, Gionotti Filho O, Ferreira TMS, Carlini EA. 1986. Pharmacology of lemongrass (Cymbopogon citratus Stapf). II. Effects of daily two month administration in male and female rats and in offspring exposed "in utero". J Ethnopharmacol 17: 65-74.

Foster HB, Niklas S, Lutz S 1980. Antispasmodic effects of some medicinal plants. Planta Med 40: 309-319.

Fratini M, Aurelio VJW, Schvan A, Batista JL, Sobral M, Schapoval EES, Henriques AT 1991. Atividade antimicrobiana de óleos essenciais. Caderno de Farmácia 7: 47-49.

Garcia EAC, Nascimento VT, Santos ABS 2003. Inotropic effects of extracts of Psidium guajava L. (guava) leaves on the guinea pig atrium. Braz J Med Biol Res 36: 661-668.

Garcia JN, Perez AMJ, Velasco NA 2000. Chemical composition of fennel oil, Foeniculum vulgare Miller, from Spain. J Essent oil Res 12: 159-162.

Germosen-Robineau L, Weniger B, Carballo A, Lagos-Witte S 1997. Farmacopea Vegetal Caribeña. ENDACARIBE: TRAMIL.

Ghelardini C, Galeotti N, Salvatore G, Mazzanti G 1999. Local anesthetic activity of the essential oil of Lavandula angustifolia. Planta Med 65: 700-703.

Gnan SO, Demello MT 1999. Inhibition of Staphylococcus aureus by aqueous goiaba extracts. $J$ Ethnopharmacol 68 : 103-108.

Grosvenor PW, Supriono A, Gray DO 1995. Medicinal plants from Riau Province, Sumatra, Indonesia. Part 2: antibacterial and antifungal activity. $J$ Ethnopharmacol 45: 97-111.

Gruenwald J, Brendler T, Jaenicke C 2000. PDR for Herbal Medicines (Physicians'Desk Reference). Montvale, New Jersey: Medical Economics Company.

Guevara JM, Chumpitaz J, Valencia E 1994. Acción in vitro de plantas sobre Vibrio cholerae. Revista de Gastroenterologia del Peru 14: 27-31.

Gugliucci A, Menini T 2002a. The botanical extracts of Achyrocline satureioides and Ilex paraguariensis prevent methylglyoxal-induced inhibition of plasminogen and antithrombin II. Life Sci 72: 279292.

Gugliucci A, Menini T 2002b. Three different pathways for human LDL oxidation are inhibited in vitro by water extracts of the medicinal herb Achyrocline satureoides. Life Sci 71: 693-705.

Gutkind GO, Martino V, Graña N, Coussio JD, Torres RA 1981. Screening of south American plants for biological activities. 1. Antibacterial and antifungal activity. Fitoterapia 213-218.

Hikino H, Takahashi M, Murakami M 1986. Isolation and hypoglycemic activity of Arborans A and B, glycans of Aloe arborescens var. natalensis leaves. Int $J$ Crude Drug Res 24: 183-186.

Himura-Lima CA, Guimarães EM, Santos CM, Di Stasi LC 2002. Commelinidae medicinais. In: Di Stasi LC, Hiruma-Lima CA (org.) Plantas medicinais na Amazônia e na Mata Atlântica. 2.ed. São Paulo: UNESP, p.41-63.

Holtez FB, Pessini GL, Sanches NR, Cortez DAG, Nakamura CV, Dias Filho BP 2002. Screening of some plants used in the Brazilian folk medicine for the tratment of infectious diseases. Mem I Oswaldo Cruz 97: 1027-1031.

Huang Y, Wang G, Chen C, Hong C, Yang MCM 1995. Fructus aurantii reduced portal pressure in portal hypertensive rats. Life Sci 57: 2011-2020.

Hyun KD, Jeong SM, Ah BE, Joo HM 2000. Inhibitory effect of herbal medicines on rotavirus infectivity. Biol Pharm Bull 23: 356-358.

Jaiarj P, Khoohaswan P, Wongkrajang Y, Peungvicha P, Suriyawong P, Saraya MLS, Ruangsonboom O 1999. A nticough and antimicrobial activities of Psidium guajava Linn. leaf extract. J Ethnopharmacol 67: 203-212.

Kadarian C, Broussalis AM, Mino J, Lopez P, Gorzalczany S, Ferraro G, Acevedo C 2002. Hepatoprotective actiovity of Achyrocline satureoides (Lam) D.C. Pharmacol Res 45: 57-61.

Kaddu S, Kerl HMD, Wolf P 2001. Accidental bullous phototoxic reactions to bergamot aromatherapy oil. J Am Acad Dermatol 45: 458-461.

Kaloga MR, Hänsel EM, Cybulski EM 1983. Isolierung eines kawapyrons aus Achyrocline satureioides. Planta Med 48: 103-104.

Kasonia K 1995. Preliminary screening of plant extracts used in respiratory pathology in Kivu Zaire on isolated guinea pigs rings trachea. Belg J Bot 128: 165-175.

Kelecon A 1983. Isolation, structure determination, and absolute configuration of barbatusol; a new bioactive diterpene with a rearranged abietane skeleton from the Labiate Coleus barbatus. Tetrahedon 39: 36033608.

Klepacz S, Frochtengarten ML, Leite JR, Calil HM 1986. Avaliação pré-clínica do eventual efeito hipnótico de Cymbopogon citratus Stapf In: Brasil, Ministério da Previdência e Assistência Social, Central de Medicamentos (org.) Considerações gerais sobre o uso do capim-cidró (Cymbopogon citratus (DC.) Stapf) em medicina popular. Brasília: CEME, p.3440,

Kuster RM, Rocha LM 2003. Cumarinas, cromonas e xantonas. In: Simões CMO, Shenkel EP, Gosmann G, Mello JCP, Mentz LA, Petrovick PR (org.) Farmacognosia: da planta ao medicamento. 5.ed. Porto Alegre/ Florianópolis: Editora da UFRGS/Editora da UFSC, p.247-262.

Kuzuya H, Tamai I, Beppu H, Shimpo K, Chihara T 2001. Determination of aloenin, barbaloin and isobarbaloin in Aloe species by micellar electrokinetic chromatography. J Chromatogr B 752: 91-97.

Langeloh A, Schenkel E 1985. Atividade antiespasmódica do extrato alcoólico de marcela (Achyrocline satureoides (Lam.) DC., Compositae) sobre a musculatura lisa genital de ratos. Caderno de Farmácia 1:38-44.

Lapa AJ, Souccar C, Lima-Landman MTR, Godinho RO, Nogueira TCML 2003. Farmacologia e toxicologia de produtos naturais. In: Simões CMO, Shenkel EP, Gosmann G, Mello JCP, Mentz LA, Petrovick PR (org.) Farmacognosia: da planta ao medicamento. 5.ed. Porto Alegre/Florianópolis: Editora da UFRGS/Editora da UFSC, p.247-262.

Lee MH, Nishimoto S, Yang LL, Yen KY, Hanto T, Yoshida T, 
Okuda T 1997. Two macrocyclic hydrolysable tannin dimers from Eugenia uniflora. Phytochemistry 44: 1343-1349.

Leite JR, Seabra MLV, Maluf E, Assolant K, Suchecki D, Tufik S, Klepacz S, Calil HM, Carlini EA 1986. Pharmacology of lemongrass (Cymbopogon citratus Stapf). III. Assessment of eventual toxic, hypnotic and anxiolytic effects on humans. J Ethnopharmacol 17: 75-83.

Lin J, Puckree T, Mvelase TP 2002. Anti-diarrhoeal evaluation of some medicinal plants used by Zulu traditional healers. J Ethnopharmacol 79: 53-56.

Lorenzetti B, Souza GEP, Sarti SJ, Santos, D, Ferreira SH 1991. Myrcene mimics the peripheral analgesic activity of lemongrass tea. $J$ Ethnopharmacol 34: 43-48.

Lorenzo D, Atti-Serafini L, Santos AC, Frizzo CD, Paroul N, Paz D, Dellacassa E, Moyna P 2000. Achyrocline satureioides essential oils from southern Brazil and Uruguay. Planta Med 66: 476-477.

Lozoya X, Morales HR, Soto MAC, García MCM, Gonzalez YL, Doubova SV 2002. Intestinal anti-spasmodic effect of a phytodrug of Psidium guajava folia in the treatment of acute diarrheic disease. $J$ Ethnopharmacol 83: 19-24.

Lutterodt GD 1992. Inhibition of Microlax-induced experimental diarrhoea with narcotic-like extracts of Psidium guajava leaf in rats. J Ethnopharmacol 37: 151157.

Lutterodt GD, Maleque A 1988. Effects on mice locomotor activity of a narcotic-like principle from Psidium guajava leaves. J Ethnopharmacol 24: 219-231.

Malini T, Vanithakumari G, Megala N, Anusya S, Devi K, Elango V 1985. Effect of Foeniculum vulgare Mill. seed extract the genital organs of male and female rats. Indian Jounal Physiological Pharmacology 29: 21-26.

MalufE, Assolante KRM, Suchecki D, Tufik S 1986. Toxicologia clínica do capim-cidrão (Cymbopogon citratus Stapf). In: Brasil, Ministério da Previdência e Assistência Social, Central de Medicamentos (org.) Considerações gerais sobre o uso do capim-cidró (Cymbopogon citratus (DC.) Stapf) em medicina popular. Brasília: CEME, p. 46-49.

Marodin SM 2000. Plantas utilizadas como medicinais no municipio de Dom Pedro de Alcântara, Rio Grande do Sul. Porto Alegre, 413p. Dissertação de Mestrado - Programa de Pós-Graduação em Botânica, Universidade Federal do Rio Grande do Sul.

Marodin SM, Baptista LRM, Mentz LA 2003. Plantas utilizadas na medicina popular do município de Dom Pedro de Alcântara, Rio Grande do Sul, Brasil. Porto Alegre: Editora da UFRGS.

Martinez MJ, Molina N, Boucourt E 1997. Evaluación de la actividad antimicrobiana del Psidium guajava L. (Guayaba). Revista Cubana de Plantas Medicinais 2: $12-14$.

Matouschek BV, Stahl-Biskup E 1991. Phytochemische untersuchung der nichflüchtigen inhaltsstoffe von Cymbopogon citratus (DC.) Stapf (Poaceae). Pharm Acta Helv 66.

Matos FJA, Viana GSB, Bandeira MAM 2001. Guia fitoterápico. Programa Estadual de Fitoterapia.Ceará: Fortaleza, Os Autores.
Matu EN, Staden J 2003. Antibacterial and anti-inflammatory activities of some plants used for medicinal purposes in Kenya.J Ethnopharmacol 87: 35-41.

Meckes M, Calzada F, Tortoriello J, González JL, Martínez M 1996. Terpenoids isolated from Psidium guajava hexane extract with depressant activity on central nervous system. Phytother Res 10: 600-603.

Mengue SS, Mentz LA, Schenkel EP 2001. Uso de plantas medicinais na gravidez. Rev Bras Farmacogn 11: 21-35.

Mesquita AAL, Correa DB, Padua AP, Guedes MLO, Gottlieb OR 1986. Flavonoids from four Compositae species. Phytochemistry 25: 1255-1256.

Ministério da Saúde 2001. Proposta de política nacional de plantas medicinais e medicamentos fitoterápicos. Brasília.

Mota TMB 1963. Substâncias antibióticas em vegetais superiores. Santa Maria. 163p. Tese para concorrer ao título de docente livre da cátedra de Microbiologia - Faculdade de Farmácia, Universidade Federal de Santa Maria.

Müller SO, Eckert I, Lutz WK, Stopper H 1996. Genotoxicity of the laxative drug components emodin, aloe-emodin and danthron in mammalian cells: Topoisomerase Imediated. Mut Res 371: 165-173.

Newall CA, Anderson LA, Phillipson JD 2002. Plantas medicinais: Guia para profissional da saúde. Londres: Editorial Premier.

Oktay M, Gülcin I, Küfrevioglu OI 2003. Determination of in vitro antioxidant activity of fennel (Foeniculum vulgare) seed extracts. Lebensm.-Wiss. U.-Technol. 36: 263-271.

Okuda T, Yoshida T, Hatano T, Yazaki K, Ashida M 1982. Ellagitannins of the Casuarinaceae, Stachyuraceae and Myrtaceae. Phytochemistry 21: 2871-2874.

Olajide OA, Awe SO, Makinde JM 1999. Pharmacological studies on the leaf of Psidium guajava. Fitoterapia 70: 25-31.

Olaniyi AA, Sofowora EA, Oguntimehin BO 1975. Phytochemical investigation of some Nigerian plants used against fevers. II. Cymbopogon citratus. Planta Med.28: 186-189.

Onawumni GO, Yisak W, Ogunlana EO 1984. Antibacterial constituents in the essential oil of Cymbopogon citratus (DC.) Stapf. J Ethnopharmacol 12: 279286.

Ozbek H, Ugras S, Dulger H, Bayram I, Tuncer I, Ozturk G, Ozturk A 2003. Hepatoprotective effect of Foeniculum vulgare essential oil. Fitoterapia 74: 317-319.

Petrovick PR, Ortega GG, Bassani V 1997. From a medicinal plant to a pharmaceutical dosage form. A (Still) long way for the Brazilian medicinal plants. Ciencia e Natura 49: 364-369.

Phillips O, Gentry AH 1993. The useful plants of Tambopata, Peru: I. Statistical hypotheses tests with a new quantitative technique. Econ Bot 47: 15-32.

Puhlmann J, Knaus U, Tubaro L, Schaefer W, Wagner H 1992. Immunubiologically active metallic ion-containing polysaccharides of Achyrocline satureioides. Phytochemistry 31: 2617-2621.

Quing MC, Teruaki A, Masao H, Kyoichi K, Tsuneo N 1991. Isolation of a human intestinal bacterium capable 
of transforming barbalion to Aloe-emodin anthrone. Planta Med 57: 15-19.

Rates SMK 2001. Promoção do uso racional de fitoterápicos: uma abordagem no ensino de Farmacognosia. Rev Bras Farmacogn 11: 57-69.

Ritter MR, Sobierajski GR, Schenkel EP, Mentz LA 2002. Plantas usadas como medicinais no município de Ipê, RS, Brasil. Rev Bras Farmacogn 12: 51-62.

Ruberto G, Baratta MT, Deans SG, Dorman HJD 2000. Antioxidant and antimicrobial activity of Foeniculum vulgare and Crithmum maritimum essential oils. Planta Med 66: 687-693.

Santos ALG 1996. Estudo tecnológico e biológico de soluções extrativas aquosas de Achyrocline satureioides (Lam.) DC., Asteraceae (marcela). Porto Alegre, 135p. Dissertação de mestrado - Faculdade de Farmácia, Universidade Federal do Rio Grande do Sul.

Santos SC, Mello JCP 2003. Taninos. In: Simões CMO, Shenkel EP, Gosmann G, Mello JCP, Mentz LA, Petrovick PR (org.) Farmacognosia: da planta ao medicamento. 5.ed. Porto Alegre/Florianópolis: Editora da UFRGS/Editora da UFSC, p.615-656.

Schapoval EES, Silveira SM, Miranda ML, Alice CB, Henriques AT 1994. Evaluation of some pharmacological activities of Eugenia uniflora L. J Ethnopharmacol 44: 137-142.

Schmeda-Hirschmann G, Theoduloz C, Franco L, Ferro E, Arias AR 1987. Preliminary pharmacological studies on Eugenia uniflora leaves: xanthine oxidase inhibitory activity. J Ethnopharmacol 21: 183-186.

Schmeda-Hirschmann G 1995. Flavonoids from Calycorectes, Campomanesia, Eugenia and Hexachlamys species. Fitoterapia 64: 373-374.

Seabra MLV, Silveira-Filho NG, Leite JR, Giraldi SMV 1986. Determinação do possível efeito ansiolítico do 'abafado' de capim-cidrão em animais de laboratório e em voluntários normais. In: Brasil, Ministério da Previdência e Assistência Social, Central de Medicamentos (org.) Considerações gerais sobre o uso do capim-cidró (Cymbopogon citratus (DC.) Stapf) em medicina popular. Brasília: CEME, p.4145.

Shah AH, Qureshi S, Ageel AM 1991. Toxicity studies in mice of ethanol extracts of Foeniculum vulgare fruit and Ruta chalepensis aerial parts. $J$ Ethnopharmacol 34: 167-172.

Shaheen HM, Ali BH, Alqarawi AA, Bashir AK 2000. Effect of Psidium gujava leaves on some aspects of the central nervours system in mice. Phytother Res 14: 107-111.

Silva LF 1993. Estudo da potência antiespasmódica de extratos hidroetanólicos $80 \%(\mathrm{v} / \mathrm{v})$ de Achyrocline satureioides (Lam.) DC. e Achyrocline vauthieriana DC. Asteraceae (marcela). Porto Alegre. 124p. Dissertação de Mestrado - Faculdade de Farmácia, Universidade Federal do Rio Grande do Sul.

Simões CMO 1984. Investigação químico-farmacológica de Achyrocline satureioides (Lam.) DC., Compositae (marcela). Porto Alegre, 186p. Tese de Mestrado -Faculdade de Farmácia, Universidade Federal do Rio Grande do Sul.

Simões CMO, Rech N, Lapa AJ 1986. Investigação farmacológica do extrato aquoso de folhas/caules de Achyrocline satureioides (Lam.) DC., Compositae (marcelas). Caderno de Farmácia 2: 37-54.

Simões CMO 1988. Antiinflammatory action of Achyrocline satureioides extracts applied topically. Fitoterapia 54: 419-421.

Simões CMO, Schenkel EP, Bauer L, Langeloh A 1988. Pharmacological investigations on Achyrocline satureioides (Lam.) DC., Compositae. J Ethnopharmacol 22: 281-293.

Simões CMO 1992. Éstude de l'activité antivirale et des relations structure-activité de substances d'orgine naturelle: plantes utilisées en medicine tradicionalle au sud du Brésil. Sapogénines et saponosides triterpeniques. França, 294p. Tese de Doutorado Univ. de Rennes.

Simões CMO, Shenkel EP, Gosmann G, Mello JCP, Mentz LA, Petrovick PR. Farmacognosia: da planta ao medicamento. 5.ed. Porto Alegre/Florianópolis: Editora da UFRGS/Editora da UFSC, p.615-656.

Sousa MP, Matos MEO, Matos FJA, Machado MIL, Craveiro AA 1991. Constituintes químicos ativos de plantas medicinais brasileiras. Fortaleza: Editora da UFC.

Souza KCB 2002. Avaliação biológica de preparações obtidas a partir das inflorescências de Achyrocline satureioides (Lam.) D.C. (Marcela). Porto Alegre, 248p. Tese de Doutorado - Curso de Pós-Graduação em Ciências Farmacêuticas, Universidade Federal do Rio Grande do Sul.

Tadon JS, Roy R, Balachandran S, Vishwakarma RA 1992. Epi-deoxycoleonol, a new antihypertensive labdane diterpenoid from Coleus forskohlii. Bioorg Med Chem Lett 2: 249-254.

Tanira MOM, Shah AH, Mohsin A, Ageel AM, Qureshi S 1996. Pharmacological and toxicological investigations on Foeniculum vulgare dried fruit extract in experimental animals. Phytother Res 10: 33-36.

Tkachenko KG, Dazarinova NV, Muzychenko LM, Shurgaya AM, Pavlova OV, Safonova NG 1999. Sanation properties of essential oils of some plant species. Rastitelnye-Resursy 35: 11-24.

Tomoyuki O, Masakazu K, Yoshio Y, Shigeyoshi I, Toshiro Y, Shoji M, Tadashi K, Kei K, Jiro I 2003. Antimicrobial activity of essential oils against Heliobacter pylori. Heliobacter 8: 207-215.

Tona L, Kambu K, Nginbi N, Cimanga K, Vlietinck AJ 1998. Antiamoebic and phytochemical screening of some Congolese medicinal plants. J Ethnopharmacol 61: 57-65.

Tyler VE 1994. Herbs of Choice - The therapeutic use of phytomedicinals. Binghamton, NY: The Haworth Press.

Vekiari SA, Protopapadakis EE, Papadopulou P, Papanicolaou D, Panou C, Vamvakias M 2002. Composition and seasonal variation of the essential oil from leaves and peel of a Cretan lemon variety. J Agric Food Chem 50: 147-153.

Vendruscolo GS 2004. Estudo das plantas utilizadas como medicinais por moradores do bairro Ponta Grossa, Rio Grande do Sul. Porto Alegre, 276p. Dissertação de Mestrado - Programa de Pós-Graduação em Botânica, Porto Alegre, Universidade Federal do Rio Grande do Sul. 
Viana GSB, Vale TG, Pinho RSN, Matos FJA 2000. Antinociceptive effect on the essential oil from Cymbopogon citratus in mice. $J$ Ethnopharmacol 70: 323-327.

Vieira RHSF, Rodrigues DP, Gonçalves SA, Menezes FGR, Aragão JS, Sousa OV 2001. Microbicidal effect of medicinal plant extracts (Psidium guajava Linn. and Carica papaya Linn.) upon bacteria isolated from fish muscle and known to induce diarrhea in children. Rev Inst Med Trop S Paulo 43: 145-148.

Vinitketkumnuen U, Puatanachokchai R, Kongtawelert P, Lertprasertsuke N, Matsushima T 1994. Antimutagenicity of lemon grass (Cymbopogon citratus Stapf) to various known mutagens in salmonella mutation assay. Mut Res 341: 1-75.

Wagner VH, Proksch A, Riess-Maurer I, Vollmar A, Odenthal S, Stuppner H, Jurcie K, Turdu ML, Fang JN 1985. Immunstimulierend wirkende polysaccharide (heteroglykane) aus höheren pflanzen. Arzneim Forsch 35: 1069-1075.

Wannissorn B, Jarikasem S, Soontorntanasart T 1996. Antifungal activity of lemon grass oil and lemon grass oil crem. Phytopher Res 10: 551-554.

Wazlawik E, Silva MA, Peters RR, Correia JFG, Farias MR, Calixto JB, Ribeiro-do-Vale RM 1997. Analysis of the role of nitric oxide in the relaxant effect on the crude extract and fractions from Eugenia uniflora in the rat thoracic aorta. J Pharm Pharmacol 49: 433-437.

World Health Organization (WHO). Traditional medicien strategy 2002-2005. 2002. Genova: World Health Organization.

Wilson CW, Shaw PE 1978. Terpene hydrocarbons from Psidium guajava. Phytochemistry 17: 1435-1436.

Wozniewski T, Blaschek W, Franz G 1990. Isolation and structure analysis of a glucamannan from the leaves of Aloe arborescens var. Miller. Carbohydr Res 198: 387-391.

Yunes RA, Pedrosa RC, Chechinel Filho V 2001. Fármacos e fitoterápicos: a necessidade do desenvolvimento da indústria de fitoterápicos e fitofármacos no Brasil. Quim Nova 24: 147-152.

Zhu M, Wong YK, Li RC 1999. Effect of oral administration of Fennel (Foeniculum vulgare) on ciprofloxacin absorption and dispo sition in the rat. J Pharm Pharmacol 51: 1391-1396. 